\title{
Atividade física em uma amostra probabilística da população do Município do Rio de Janeiro
}

\author{
Physical activity in a probabilistic sample \\ in the city of Rio de J aneiro
}

Valéria Barbosa Gomes 1

Kamile Santos Siqueira 1

Rosely Sichieri 1

\footnotetext{
1 Departamento de Epidemi ologia, Universidade do Estado do Rio de Janeiro. Rua São Francisco Xavier 524, 7o andar, Bloco E, Rio de Janeiro, RJ 20550-012, Brasil. sichieri@uerj.br
}

Abstract This study evaluated physical activity in a probabilistic sample of 4,331 individuals 12 years of age and older resi ding in the city of Rio de Janeiro, who participated in a household survey in 1996. Occupation and leisure activity were grouped according to categories of energy expenditure. The study also eval uated number of hours watching TV, using the computer, or playing video-games. Only $3.6 \%$ of males and $0.3 \%$ of females reported heavy occupational work. A full $59.8 \%$ of males and $77.8 \%$ of females reported never performing recreati onal physical activity, and there was an increase in this prevalence with age, especially for men. Women's lei sure activities involved less energy expenditure and had a lower median duration than those of men. Mean daily TV/video/computer time was greater for women than for men. The greater the level of schooling, the higher the frequency of physical activity for both sexes. Analyzed jointly, these data show the low energy expenditure through physical activity by the population of the city of Rio de Janeiro. Women, the middle-aged, the elderly, and low-income individuals were at greatest risk of not performing recreational physical activity.

Key words Physical Exercise; Energy Metabolism; Leisure Activities

Resumo O presente estudo avaliou a atividade física em uma amostra probabilística de 4.331 indivíduos com 12 anos ou mais, moradores no Município do Rio deJaneiro, que participaram de um inquérito domiciliar em 1996. Ocupação elazer foram agrupados segundo categori as de gasto energético. Horas assistindo tel evi são ou uti lizando computador ou vi deo game foram também avaliadas. Somente 3,6\% dos homens e 0,3\% das mulheres referi ram ocupação pesada. Entre os homens 59,8\% referi ram que nunca realizavam atividade física de lazer e entre as muIheres este percentual foi de $77,8 \%$, ocorrendo um importanteaumento desta prevalência com a idade, principalmente para homens. Mulheres realizam atividades delazer de menor gasto energético do que os homens e com du ração mediana também menor. Para horas assistindo tel evisão/vídeo/computador a média diária foi maior para as mulheres do que para os homens. Quanto mai or o grau de escolaridade, maior a freqüência de atividadefísica de lazer em ambos os sexos. Analisa dos em conj unto, estes dados mostram o baixo gasto energéti co da população do Município do Rio de Janeiro com atividadefísica, sendo que as mulheres, os grupos de meia idade idosos e os de baixa escolaridade apresentam um mai or risco de não realizar atividade física delazer.

Palavras-chave Exercício Físico; Metabolismo Energético; Atividades de Lazer 
Introdução

A atividade física é operacionalmente definida como os movimentos corpóreos produzidos pel os músculos esqueléticos que resultam em gasto energético. Estima-se que $15 \%$ a $40 \%$ do gasto energético total (Bouchard et al., 1993), e mais de $50 \%$ da variação de gasto energético entre populações (Willett, 1998), associa-se à atividade física.

Realizar exercícios regularmente é um dos poucos fatores que podem prevenir o ganho de peso. Adicionalmente, o condicionamento físico obtido através do exercício, reduz a mortalidade e a morbidade, mesmo nos indivíduos que se mantêm obesos (Jebb \& Moore, 1999; McInnis, 2000; WHO, 1997). Estima-se que pequenos aumentos de atividade física em populações sedentárias, teriam um impacto maior na redução das doenças crônicas do que a redução do tabagismo (U. S. Preventive Service Task Force, 1996). Este impacto decorre do fato de que o sedentarismo associa-se a várias doenças e condições metabólicas adversas como: obesidade, doença coronariana, hipertensão, diabetes tipo 2, osteoporose, câncer de cólon, depressão (Bouchard, 1996), perfil lipídico e tolerância à glicose (Duncan et al., 1991; Kohl et al., 1992).

Embora sua importância na manutenção da saúde, a atividade física tem se reduzido muito nas sociedades modernas, principalmente nos grupos de menor nível sócio-econômico. Nos países desenvolvidos, a maioria das ocupações são de baixo gasto energético e as atividades físicas associadas ao lazer diferenciam os grupos mais ativos dos menos ativos. Nos Estados Unidos, em 1985, 56\% dos homens e $61 \%$ das mul heres não realizavam nenhuma atividade física de lazer, não tendo havido grande variação destes percentuais na década de 90 (Kriska \& Caspersen, 1997). No mais recente inquérito de âmbito nacional americano (NHANESIII - Third National Heal th and Nutrition Examination Survey, 1988-1994) sedentarismo foi mais freqüente entre as minorias étnicas e raciais. Entre a população branca 18\% referiram não fazer atividade física no tempo de lazer, contra $35 \%$ dos afro-americanos e $40 \%$ dos hispano-americanos. Indicadores de classe social não explicaram as diferenças observadas segundo grupo étnico, no referido estudo, tendo sido sugerido que outras variáveis como suporte social, segurança e barreiras ambientais deveriam ser mais exploradas na explicação da inatividade (Crespo et al., 2000).

Diferentes estudos também mostram que o sedentarismo é mais freqüente entre as mulhe- res, os idosos e nos indivíduos com menor nível de escolaridade (Crespo et al., 2000; M isigojDurakovic et al., 2000; M MWR, 2000). A associação entre nível sócio-econômico e atividade física é contudo complexa. Assim, praticar esportes, em estudo realizado na Croácia, associou-se ao nível educacional, mas o tempo gasto em atividade física de lazer não associou-se à escolaridade (Misigoj-Durakovic et al., 2000).

Vários são os componentes da atividade física diária e medi-la não é uma tarefa fácil. Vários questionários (Kriska \& Caspersen, 1997) e equipamentos (Kriska, 2000) foram desenvolvidos e validados, mas a sua utilização depende muito do desenlace a ser associado à atividade física. Para avaliação de prevalência de atividade física na população, os componentes mais utilizados são a ocupação e a atividade física de lazer. Adicionalmente, horas assistindo televisão têm sido utilizadas como indicador de sedentarismo (Grund et al., 2000).

Um vez que dados de base populacional sobre atividade física no Brasil são escassos, o objetivo deste estudo foi de descrever três marcadores de atividade física: ocupação, lazer e horas assistindo tel evisão, em uma amostra probabilística de adolescentes, adultos e idosos do Município do Rio de Janeiro.

\section{Metodologia}

O estudo que originou os dados da presente análise foi desenvolvido para avaliar a prevalência de obesidade e seus dois gran des determinantes: o consumo alimentar e a atividade física, no Município do Rio de Janeiro em 1996. Os domicílios foram selecionados por amostragem probabilística, por conglomerados em duas etapas. Sorteou-se sessenta setores censitários dos setores da Pesquisa Nacional de Amostragem Domiciliar (PNAD) de 1995, e nestes 34 domicílios foram sorteados. O cálculo da amostra tomou por base prevalências de obesidade da ordem de $15 \%$ entre adolescentes, $40 \%$ entre os adultos e $30 \%$ entre os idosos, verificando-se que uma amostra composta por, no mínimo 1.632 domicílios ( $80 \%$ dos 2.040 iniciais) asseguraria, em todos os domínios de estudo, a obtenção de estimativas para proporções sob níveis de precisão máximos iguais a $5 \%$ (Sichieri, 1998). Para atividade física considerando-se uma prevalência de $50 \%$, a precisão seria de 0,03 entre os idosos e de 0,01 entre adultos, conforme a fórmula:

$$
\sqrt{1,96 \cdot \frac{p \cdot(1-p)}{(n-1)}}
$$


Dos domicílios sorteados 83,2 participaram da pesquisa. A taxa de não resposta foi variável para os diferentes segmentos do questionário, sendo que dos 4.551 indivíduos na faixa etária de 12 anos ou mais, 4.331 (95,2\%) responderam as questões relativas à ocupação e 4.307 responderam a questão relativa a horas assistindo televisão $(94,6 \%)$. As análises univariadas utilizaram a totalidade das respostas, o que faz com que os totais das diferentes tabel as sejam diferentes.

A atividade física foi avaliada através de questionário contendo informações, referentes ao mês anterior, sobre ocu pação, deslocamento para o trabal ho e escola, cuidados com criança menor de três anos e atividade física de lazer. Avaliou-se, também, horas assistindo televisão, vídeo, video game ou em atividades em computador. Esta variável será referida no texto como horas assistindo tel evisão e o questionário encontra-se na Figura 1 . A atividade física de lazer foi aval iada pelas questões 7 e 8. Para a questão 8 considerou-se resposta afirmativa, qual quer resposta relativa à quantificação da duração e considerou-se como não resposta a incapacidade de informar a duração da atividade.

As ocupações foram categorizadas segundo graus crescentes de gasto energético, conforme Food and Agriculture Organization/World Health Organization/United Nations University (FAO/WHO/UNU, 1985) e a Portaria 3.214/ 78 do Ministério do Trabalho (1978). Considerouse como atividade leve as ocupações exercidas sentadas, com movimentos leves de braços e tronco; em pé, com trabal ho leve em máquina ou bancada movimentando braços e pernas como por exemplo: médico, advogado, bancário, operador de caixa, motorista, auxiliar de escritório, balconista, professor, vendedor, com gasto energético entre 125 e $150 \mathrm{Kcal} / \mathrm{h}$; atividade moderada as ocupações exercidas de pé, com trabalho leve em máquinas ou bancada com movimentação vigorosa de braços e pernas e ocupações exercidas de pé, como trabaIho moderado em máquina ou bancada, com movimentação vigorosa de braços e as ocupações exercidas em movimento, como trabalho moderado de levantar ou empurrar, com gasto energético entre 175 e $300 \mathrm{Kcal} / \mathrm{h}$, como por exemplo: carteiro, contínuo, vendedor domiciliar, pintor de parede, eletricista, marceneiro, mecânico de automóveis, faxineiro, caseiro e considerou-se como atividade pesada, o trabaIho intermitente de levantar ou arrastar, com gasto energético entre 440 a $550 \mathrm{Kcal} / \mathrm{h}$, como por exemplo: servente de pedreiro, lixeiro e estivador. Quando mais de uma ocupação foi re-
Figura 1

Modelo de questionário sobre atividade física para todos os moradores com 12 anos ou mais.

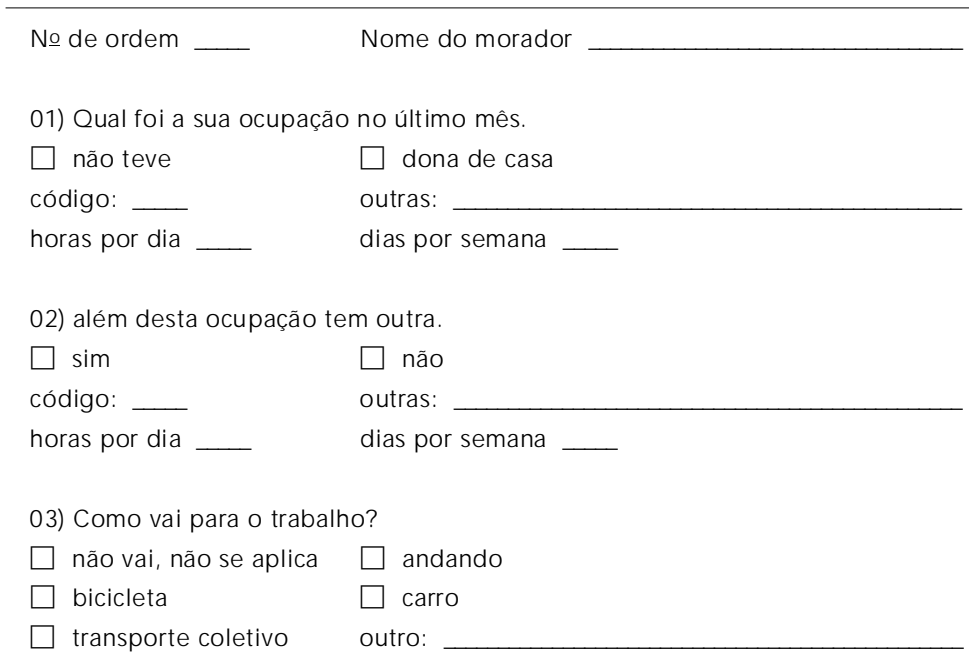

04) Se vai andando, de bicicleta ou outro meio que dispenda energia (patins, etc...), quanto tempo gasta por dia somando ida e volta. minutos por dia

05) Q uantas horas assiste TV ou vídeo ou videogame/co mputador.

Não assiste horas por dia horas por semana

06) Toma conta de crianças menores de 3 anos?

$\square \operatorname{sim} \quad \square$ não vezes por semana __ horas por dia

07) Faz atividade física regular ou esportes:

$\square$ nunca ou quase nunca $\square$ algumas vezes $\square$ sempre

08) Pratica atividade física, além da lo comoção para o trabalho e escola.

$\square \operatorname{sim} \quad \square$ não

Se sim, preencha o quadro a seguir. (Se mais de uma atividade do mesmo grupo, some freqüência e duração).

\begin{tabular}{|c|c|c|}
\hline Grupos & freqüência & Duração em minutos \\
\hline I - Caminhadas & $\begin{array}{l}\text { pordia } \\
\text { por semana }\end{array}$ & 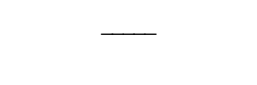 \\
\hline $\begin{array}{l}\text { Il - Andar em alta velocidade } \\
\text { Correr, bicicleta, vôlei, ginástica, } \\
\text { dança, musculação, pegar onda }\end{array}$ & $\begin{array}{l}\text { por dia } \\
\text { p porsemana }\end{array}$ & 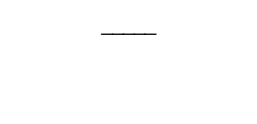 \\
\hline $\begin{array}{l}\text { III - Natação, futebol, alpinismo, } \\
\text { basquete }\end{array}$ & $\begin{array}{l}\text { pordia } \\
\text { por semana }\end{array}$ & 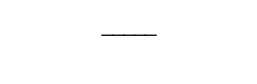 \\
\hline O utros: Q uais & $\begin{array}{l}\text { por dia } \\
\ldots \text { por semana }\end{array}$ & 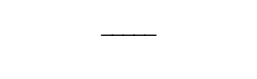 \\
\hline
\end{tabular}


ferida, computou-se a ocupação com maior gasto energético. Escolaridade foi categorizada em: 5 anos ou menos, 6 a 9 anos e 10 anos ou mais de educação.

As prevalências foram calculadas levandose em conta o desenho da amostra por conglomerados. Para o cálculo do erro padrão, bem como para as análises estatísticas, utilizou-se o programa Software for Statistical Analysis for Correlated Data - SUDAAN (Research Triangle Institute, 1991). As comparações segundo sexo, foram realizadas através do testet de Student, ou do qui-quadrado. Para a comparação entre os três níveis de escolaridade utilizou-se a análise de variância, e a análise dos fatores associados à realização de atividade física de lazer, tratada como variável dicotômica, foi feita através de regressão logística.

\section{Resultados}

Quanto à ocupação, a atividade considerada pesada foi praticada por somente $3,6 \%$ dos homens e $0,3 \%$ das mulheres. Entre as mulheres ocupações de alto gasto energético foram de menor prevalência do que entre homens, em todas as faixas de idade (Tabela 1 ).

Realizar sempre alguma atividade de lazer regular ou esporte (questão 7) foi referida por $18,4 \%$ dos homens e $9,1 \%$ das mulheres (Tabela 2). Em todas as faixas etárias as mulheres realizam atividade física de lazer ou esporte com menor freqüência. Estes resultados foram próximos aos obtidos com a questão mais restrita

Tabela 1

Tamanho da amostra e ocupação no mês anterior, classificada segundo gasto energético*. Pesquisa de Nutrição e Saúde, Município do Rio de Janeiro, 1996.

\begin{tabular}{lccccc}
\hline $\begin{array}{l}\text { Grupo de } \\
\text { idade (anos) }\end{array}$ & $\mathrm{n}$ & \multicolumn{4}{c}{ Atividade segundo ocupação $\%$} \\
& & Sem ocupação** & Leve & Moderada & Pesada \\
\hline Homens & & & & \\
$12-20$ & 410 & 73,7 & 19,2 & 4,9 & 2,1 \\
$21-40$ & 743 & 19,7 & 61,3 & 13,6 & 5,3 \\
$41-60$ & 538 & 31,4 & 51,6 & 13,7 & 3,3 \\
$61-91$ & 273 & 76,2 & 14,5 & 7,4 & 1,8 \\
Total & 1.964 & 42,2 & 43,2 & 10,9 & 3,6 \\
Mulheres & & & & & \\
$12-20$ & 377 & 84,3 & 13,8 & 1,8 & - \\
$21-40$ & 920 & 54,1 & 40,1 & 5,4 & 0,4 \\
$41-60$ & 652 & 68,1 & 26,7 & 4,9 & 0,3 \\
$61-98$ & 418 & 93,4 & 5,4 & 0,7 & - \\
Total & 2.367 & 69,8 & 26,0 & 3,9 & 0,3 \\
\hline
\end{tabular}

* FAO/WHO/UNU (1985); Ministério do Trabalho (1978).

** Inclui donas de casa, estudantes e aposentados. que requeria especificar a atividade, freqüência e duração (questão 8). A questão 8 não foi respondida por 19 homens e 26 mulheres que responderam a questão 7 (Tabela 2). Mesmo excluindo os que não responderam a questão 8, os percentuais de atividade física aferido pela pergunta 7 são, ligeiramente, maiores do que os aferidos pela 8 .

Tendo em vista que a questão 7 tem menor percentual de não resposta e que as análises baseadas nela ou na questão mais restrita (questão 8) forneceram associações similares em termos de diferenças por sexo, ocupação e escolaridade, as análises bivariadas ou multivariadas são apresentadas baseadas na questão 7 .

Mulheres realizam atividades de lazer de menor gasto energético do que os homens e com duração mediana também menor. Para os entrevistados que referiram realizar atividade física, as atividades do grupo I , conforme questionário (Figura 1), questão 8 , foram referidas por $19,0 \%$ dos homens e $46,5 \%$ das mulheres, as do grupo II, por $37,1 \%$ dos homens e $44,0 \%$ das mulheres e as do grupo III por $43,9 \%$ dos homens e por $9,5 \%$ das mulheres. A duração mediana destas atividades por semana nos grupos I, II e III foram, respectivamente, de 60, 80 e 90 minutos para os homens, e de 60, 60 e 70 minutos para as mulheres

Quanto à ocupação, homens e mulheres com ocupação moderada e pesada tendem a fazer menos atividade física de lazer do que os sem atividade ou com ocupação leve (Tabela 3).

A média diária de horas assistindo televisão foi de 3,5 para os homens e de 3,8 para as muIheres. Esta diferença por sexo foi estatisticamente significante $(p=0,01)$ só para a população total (Tabela 4). Entre as mulheres, 7,7\% não assistiam televisão e 27,9\% viam menos de duas horas por dia. Entre os homens, 9,0\% não assistiam televisão e 32,7 \% viam menos deduas horas por dia. Os adolescentes são os que mais tempo gastam nesta atividade.

Atividade física associada à locomoção para o trabalho e cuidados com criança menor de três anos, foram de baixa prevalência entre os dois sexos, com 9,6\% (SE $=0,7$ ) dos homens e $7,0(\mathrm{SE}=0,6)$ das mulheres referindo ir para o trabal ho andando ou de bicicleta. Tomar conta de criança foi tarefa referida por $4,8 \%(\mathrm{SE}=0,5)$ dos homens e 13,0 (SE $=0,7)$ das mulheres, com o maior percentual (19,5\%) entre as muIheres de 20 a 40 anos.

Quanto maior o grau de escolaridade, maior é a freqüência de atividade física de lazer nos dois sexos e menos horas são gastas assistindo televisão entre as mulheres. Observou-se, ainda, ausência de associação entre escolaridade 
e horas assistindo televisão entre os homens (Tabela 5).

Os fatores associados à realização de atividade física de lazer em análise multivariada foram: ser do sexo masculino, jovem e com maior escolaridade. Adicionalmente, mais horas assistindo tel evisão associou-se negativamente à atividade física de lazer (Tabela 6).

\section{Discussão}

Questionários de atividade física, como o utilizado neste trabalho, são freqüentemente escoIhidos para estudos em populações, pois não interferem na atividade do entrevistado, são práticos e de fácil aplicabilidade (Kriska \& Caspersen, 1997). No entanto, a mensuração da atividade física é uma tarefa complexa. Na maior parte dos estudos populacionais as estimativas restringem-se à atividade física de lazer, em função da dificuldade na obtenção de dados relacionados à ocupação e trabalhos realizados no domicílio (Caspersen \& Mathew, 1997).

Mesmo com toda dificuldade de mensuração, baixo grau de atividade física é considerado um importante fator de risco para muitas das doenças crônicas (Blair, 1997) e para obesidade (Hill, 1997). Heini \& Weinsier (1997) consideram que o estilo de vida moderno, centrado no sedentarismo, é crucial no desenvolvimento da obesidade, que por sua vez associase a várias doenças crônicas, como diabetes mellitus, hipertensão arterial e doença coronariana.

Neste estudo populacional, no Município do Rio de Janeiro, observamos que as atividades relacionadas à ocupação são predominantemente de baixo gasto energético. No Brasil, a modernização dos processos produtivos, inclusive na agricultura, característica das últimas décadas, se traduz em uma redução da atividade física (INAN, 1991).

O baixo nível de atividade física associada ao trabal ho nos centros urbanos, faz com que as atividades de lazer sejam a melhor representação das atividades físi cas da população (Kriska \& Caspersen, 1997; Powell et al., 1987).

Para o Município do Rio de Janeiro a maioria da população não faz atividade física de lazer e passa muitas horas assistindo televisão. A situação que descrevemos para Rio de Janeiro é, provavelmente, similar a de outras áreas metropolitanas, onde a preval ência de obesidade, principal mente em mulheres é crescente (Sichieri et al., 1994).

No presente estudo, os homens referiram realizar atividade física de lazer com maior fre-
Tabela 2

Percentual da população com atividade física de lazer e erro padrão (EP). Pesquisa de Nutrição e Saúde, Município do Rio de Janeiro, 1996.

\begin{tabular}{|c|c|c|c|c|c|}
\hline \multirow[t]{2}{*}{$\begin{array}{l}\text { Grupo de } \\
\text { idade (anos) }\end{array}$} & \multicolumn{5}{|c|}{$\begin{array}{c}\text { Com atividade física de lazer regular ou esporte } \\
\text { (questão 7, Figura 1) }\end{array}$} \\
\hline & $\mathrm{n}$ & Nunca & Ald & & Sempre \\
\hline \multicolumn{6}{|l|}{ Homens } \\
\hline $12-20$ & 432 & 38,6 & & & 33,4 \\
\hline $21-40$ & 773 & 56,6 & & & 18,0 \\
\hline $41-60$ & 548 & 70,1 & & & 11,1 \\
\hline $61-91$ & 276 & 80,2 & & & 11,2 \\
\hline Total & 2.029 & 59,8 & & & 18,4 \\
\hline \multicolumn{6}{|l|}{ Mulheres } \\
\hline $12-20$ & 391 & 63,4 & & & 13,8 \\
\hline $21-40$ & 948 & 79,4 & & & 7,6 \\
\hline $41-60$ & 666 & 79,9 & & & 8,6 \\
\hline $61-91$ & 423 & 83,5 & & & 9,3 \\
\hline Total & 2.428 & 77,8 & & & 9,1 \\
\hline \multirow[t]{2}{*}{$\begin{array}{l}\text { Grupo de } \\
\text { idade (anos) }\end{array}$} & \multicolumn{5}{|c|}{$\begin{array}{l}\text { Referiram duração da atividade física de lazer regular } \\
\text { (questão } 8, \text { Figura } 1 \text { ) }\end{array}$} \\
\hline & & & $\%$ & EP & \\
\hline \multicolumn{6}{|l|}{ Homens } \\
\hline $12-20$ & & & 59,6 & 2,5 & \\
\hline $21-40$ & & & 39,0 & 1,9 & \\
\hline $41-60$ & & & 24,1 & 2,0 & \\
\hline $61-98$ & & & 20,7 & 2,7 & \\
\hline Total & & & 36,7 & 1,2 & \\
\hline \multicolumn{6}{|l|}{ Mulheres } \\
\hline $12-20$ & & & 30,6 & 2,6 & \\
\hline $21-40$ & & & 17,0 & 1,4 & \\
\hline $41-60$ & & & 17,5 & 1,7 & \\
\hline $61-98$ & & & 16,4 & 2,0 & \\
\hline Total & & & 19,2 & 0,9 & \\
\hline
\end{tabular}

Tabela 3

Percentual da população com de atividade física de lazer* e erro padrão (EP), segundo ocupação**. Pesquisa de Nutrição e Saúde, Município do Rio de J aneiro, 1996.

\begin{tabular}{lcccc}
\hline $\begin{array}{l}\text { Grupo de } \\
\text { idade (anos) }\end{array}$ & $n$ & Sem ocupação*** & Leve & $\begin{array}{c}\text { Moderada } \\
\text { e Pesada }\end{array}$ \\
\hline Homens & & & & \\
$12-20$ & 410 & 65,2 & 60,1 & 39,2 \\
$21-40$ & 743 & 44,3 & 47,1 & 36,6 \\
$41-60$ & 537 & 25,1 & 33,6 & 22,5 \\
$61-91$ & 237 & 22,1 & 17,8 & 3,6 \\
Total & 1.963 & 42,8 & 42,4 & 32,0 \\
Mulheres & & & & $-* * * *$ \\
$12-20$ & 377 & 37,9 & 37,4 & 2,5 \\
$21-40$ & 920 & 17,1 & 26,1 & - \\
$41-60$ & 652 & 18,8 & 28,2 & 9,3 \\
$61-98$ & 418 & 17,6 & 3,6 & \\
Total & 2.367 & 21,6 & 26,8 & \\
\hline
\end{tabular}

* Com base nos que referiram alguma atividade na questão 7 da Figura 1.

** FAO/WHO/UNU (1985); M inistério do Trabalho (1978).

*** Inclui donas de casa, estudantes e aposentados.

$* * * * \mathrm{n}<10$. 
Tabela 4

Médias e erro padrão de horas diárias assistindo televisão/vídeo/computador, segundo faixa etária. Pesquisa de Nutrição e Saúde, Município do Rio de J aneiro, 1996.

\begin{tabular}{|c|c|c|c|c|c|c|c|}
\hline \multirow[t]{2}{*}{ Idade (anos) } & \multicolumn{3}{|c|}{ Homens } & \multicolumn{3}{|c|}{ Mulheres } & \multirow[t]{2}{*}{ P t-test } \\
\hline & $\mathrm{n}$ & Média & $\mathrm{EP}$ & $\mathrm{n}$ & Média & EP & \\
\hline 12 a 20 & 410 & 4,4 & 0,12 & 377 & 4,5 & 0,12 & 0,56 \\
\hline 21 a 40 & 742 & 3,2 & 0,07 & 914 & 3,5 & 0,07 & 0,19 \\
\hline 41 a 60 & 533 & 3,2 & 0,08 & 643 & 3,5 & 0,08 & 0,24 \\
\hline$>60$ & 273 & 3,8 & 0,14 & 415 & 4,1 & 0,11 & 0,10 \\
\hline Total & 1.958 & 3,5 & 0,05 & 2.349 & 3,8 & 0,05 & 0,01 \\
\hline
\end{tabular}

EP = Erro Padrão.

\section{Tabela 5}

Percentuais de realização regular de atividade física de lazer e médias de horas assistindo televisão/dia, segundo escolaridade. Pesquisa de N utrição e Saúde, Município do Rio de J aneiro, 1996.

\begin{tabular}{lcc}
\hline A nos de educação & $\begin{array}{c}\text { Atividade física de lazer } \\
\%\end{array}$ & \\
& & \\
\hline Homens & \\
$0-5$ & 17,8 & 2,5 \\
$6-9$ & 27,0 & 2,7 \\
10 ou mais & 39,6 & 2,1 \\
$\chi^{2}-p$ & 0,0001 & \\
Mulheres & & \\
$0-5$ & 6,1 & 1,2 \\
$6-9$ & 10,8 & 1,6 \\
10 ou mais & 24,8 & 1,8 \\
$\chi^{2-p}$ & 0,0001 & \\
\hline
\end{tabular}

A nos de educação

Horas/dia assistindo televisão/vídeo/computador Média

EP

\begin{tabular}{lll}
\hline Homens & & \\
$0-5$ & 3,5 & 0,10 \\
$6-9$ & 3,6 & 0,07 \\
10 ou mais & 3,5 & 0,14 \\
AN OVA p-valor & 0,20 & \\
Mulheres & & \\
0-5 & 3,9 & 0,09 \\
6-9 & 3,8 & 0,06 \\
10 ou mais & 3,2 & 0,12 \\
AN OVA p-valor & 0,0001 &
\end{tabular}

$\mathrm{EP}=$ Erro Padrão. qüência, eles também têm maior gasto energé tico relacionado à ocupação e assistem televisão durante menos tempo do que as mulheres, o que poderia explicar o diferencial, segundo gênero, nas prevalências de obesidade (Sichieri, 1998).

Os adolescentes são os que apresentam maior prevalência de atividade física de lazer para ambos os sexos, embora as menin as realizem menos atividade física que os meninos neste e em outros estudos (Fonseca et al., 1998). Mesmo com realização menos freqüente, meninas que referiram al guma atividade física de lazer, na população em estudo, apresentavam menor prevalência de sobrepeso 3,6 versus $15,4 \%$ (Sichieri, 1998), sugerindo ser este um importante caminho na preven ção da obesidade.

Adolescentes passam mais tempo assistindo tel evisão que outras faixas de idade. O número de horas que o adolescente passa assistindo tel evisão é um importante fator associado à obesidade. Estima-se um aumento de $2 \%$ na prevalência adicional de obesidade para cada hora adicional de televisão em jovens americanos de 12 a 17 anos (Dietz \& Gortmaker, 1985). Estudo realizado com 391 adolescentes matriculados em uma escola privada do município de Niterói, à semelhança do observado por Dietz, encontrou associação positiva entre IMC e horas assistindo televisão (Fonseca et al., 1998). No presente estudo, em todas as faixas de idade, não assistir tel evisão ou assistir por menos de duas horas, comparativamente a quem assiste mais de cinco horas por dia, associou-se positivamente à realização de alguma atividade física de lazer (Tabela 6).

Logo após a adolescência o padrão de atividade muda, reduzindo-se de forma importante, fato observado também por Heini et al. (1997) para os Estados Unidos, onde após a adolescência há um declínio nas atividades fí- 
sicas vigorosas de $42 \%$ entre os homens e de $30 \%$ entre as mulheres (Yusuf et al., 1996).

Nos Estados Unidos, 37\% dos adultos com 2o grau ou menos e 14\% dos universitários, não realizam nenhuma atividade física de lazer (Heini et al., 1997). No presente estudo, importante associação positiva também foi observada entre nível de educação e atividade física de lazer. Na analise multivariada a odds ratio para os indivíduos com mais de dez anos de escolaridade, comparados aos com até cinco anos de estudos, foi de 4,09.

Em conclusão, os resultados do presente trabalho mostraram que as taxas de realização de atividade física, nesta capital estudada, são baixas tanto no trabalho quanto no lazer. As mulheres, os grupos de meia idade e os idosos, os indivíduos de baixa escolaridade e os que assistem mais tempo de tel evisão, apresentam um maior risco de não realizarem atividades físicas de lazer. Estimular a atividade física, tanto entre homens, quanto entre mulheres, de forma acessível à toda população, seria, provavelmente, uma boa estratégi a para prevenção das doenças crônicas do adulto, particularmente da obesidade.

\section{Referências}

BLAIR, S. N., 1997. Effect of physical activity on cardiovascular disease mortality as an independent risk factor. In: The Prevalence of Physical Inactivity in the United States (A. S. Leon, ed.), pp. 127136, Champaign: Human Kinetics Books.

BOUCHARD, C., 1996. Can obesity be prevented? Nutrition Reviews, 54:S125-S130.

BOUCHARD, C.; SHEPARD, R. J. \& STEPHENS, T., 1993. Physical activity, fitness, and health. Consensus statement. In: Physical Activity and Cardiovascular Health (A. S. Leon, ed.), pp. 27-29, Champaign: Human Kinetics Books.

CASPERSEN, C. J. \& MATHEW, M. Z., 1997. Physical activity and cardiovascular health. In: The Prevalence of Physical Inactivity in the United States (A. S. Leon, ed.). pp. 32-39, Champaign: Human Kinetics Books.

CRESPO, C. J.; SMIT, E.; ANDERSEN, R. E.; CARTERPOKRAS, O. \& AINSWORTH, B. E., 2000. Race/ ethnicity, social class and their relation to physical inactivity during leisure time: Results from the Third National Health and Nutrition Examination Survey, 1988-1994. American Journal of Preventive Medicine, 18:46-53.

DIETZ, W. H. \& GORTM AKER, S. L., 1985. Do we fatten our children at the television set? Obesity and television viewing in children and adolescents. Pediatrics, 75:807-812.
Tabela 6

O dds ratio e intervalo de confiança para realização de atividade física* de lazer ou esporte. Modelo com todas as variáveis da tabela. Pesquisa de Nutrição e Saúde, Município do Rio de J aneiro, 1996.

\begin{tabular}{|c|c|c|c|}
\hline \multirow[b]{2}{*}{ Sexo masculino/feminino } & \multirow{2}{*}{$\begin{array}{c}\text { Odds ratio } \\
2,39\end{array}$} & \multicolumn{2}{|c|}{ IC $95 \%$} \\
\hline & & 2,03 & 2,81 \\
\hline \multicolumn{4}{|l|}{ Grupo de idade (anos) } \\
\hline $12-20 />60$ & 4,02 & 3,03 & 5,35 \\
\hline $21-40 />60$ & 1,46 & 1,11 & 1,92 \\
\hline $41-60 />60$ & 1,13 & 0,85 & 1,51 \\
\hline \multicolumn{4}{|l|}{ O cupação } \\
\hline Sem ocupação/moderada + pesada & 1,10 & 0,92 & 1,32 \\
\hline Leve/moderada + pesada & 0,69 & 0,35 & 1,36 \\
\hline \multicolumn{4}{|l|}{ Escolaridade } \\
\hline$\geq 10$ anos/0-5 anos & 4,09 & 3,17 & 5,28 \\
\hline 6-9anos/0-5 anos & 1,85 & 1,49 & 2,30 \\
\hline \multicolumn{4}{|l|}{ Horas assistindo televisão/vídeo/dia } \\
\hline Não assiste/ $\geq 5$ horas/dia & 1,42 & 1,04 & 1,94 \\
\hline$<2$ horas/ $\geq 5$ horas/dia & 1,56 & 1,23 & 1,98 \\
\hline $2-5$ horas $/ \geq 5$ horas/dia & 0,89 & 0,89 & 1,38 \\
\hline
\end{tabular}

* Sempre ou alguma atividade/nunca (questão 7 da Figura 1).
DUNCAN, J. J.; GORDON, N. F. \& SCOTT, C. B., 1991. Woman walking for health and fitness. How much is enough. JAMA, 266:3295-3299.

ENDEF (Estudo Nacional da Despesa Familiar), 1977. Consumo Alimentar - Antropometria. Rio de Janeiro: Fundação Instituto Brasileiro de Geografia e Estatística.

FAO (Food and Agricultural Organization)/WHO (World Health Organization)/UNU (United Nations University), 1985. Energy and Protein Requirements. Geneva:WHO.

FON SECA, V. M.; SI CHIERI, R. \& VEIGA V., 1998. Fatores associados à obesidade em adolescentes. Revista de SaúdePública, 32:541-549.

GRUND, A.; DILBA, B.; FORBERGER, K., KRAUSE, H.; SIEWERS, M.; RIECKERT, H. \& MULLER, M. J., 2000. Relationships between physical activity, physical fitness, muscle strength and nutritional state in 5- to 11-year-old children. European Journal of Applied Physiology and Occupational Physiology, 82:425-438.

HEINI, A. F. \& WEINSIER, R. L., 1997. Divergent trends in obesity and fat intake patterns: The American paradox. American Journal of Medicine, 120:259264.

HILL, J. O., 1997. Physical activity body weight and body fat distribution. In: The Preval ence of Physical Inactivity in the United States (A. S. Leon, ed.), 
pp. 88-97, Champai gn: Human Kinetics Books.

INAN (Instituto Nacional de Alimentação e Nutrição), 1991. Condições nutricionais da população brasileira: Adultos e idosos. In: Pesquisa Nacional sobreSaúde e Nutrição (Ministério da Saúde - MS, org.), p. 32, Brasília: INAN/MS.

JEBB, S. A. \& MOORE, M. S., 1999. Contribution of a sedentary lifestyle and inactivity to the etiology of overweight and obesity: Current evidence and research issues. Medicine and Science in Sports and Exercise, 31(Sup. 11):S534-541.

KOHL, H. W.; GORDON, N. F.; VILLEGAS, S. A. \& BLAIR, S. N., 1992. Cardiorespiratory fitness, glycemic status, and mortality risk in men. Diabetes Care, 15:184-192.

KRISKA, A. M., 2000. Ethnic and cultural issues in assessing physical activity. Research Quarterly for Exercise and Sport, 71(Sup. 2):S47-53.

KRISKA, A. M. \& CASPERSEN, C. J.,1997. Introduction to a collection of physical activity questionnaires. Medicine and Science in Sports and Exercise, 29 (Sup. 6):S5-9.

M cINNIS, K. J., 2000. Exercise and obesity. Coronary Artery Disease, 11:111-116.

MINISTÉRIO DO TRABALHO, 1978. Lei Número 6.514 de 22 de Dezembro de 1977. Altera o Capítulo V do Título II da Consolidação das Leis do Trabalho, Relativas à Segurança e Medicina do Trabal ho, e dá Outras Providências. São Paulo: Lex Editora.

MISIGOJ-DURAKOVIC, M.; HEIMER, S.; MATKOVIC, B. R.; RUZIC, L. \& PRSKALO, I., 2000. Physical activity of urban adult population: Questionnaire Study. Croatian Medical Journal, 15:428-432.
MMWR (Morbidity and Mortality Weekly Report), 2000. Prevalence of leisure-time and occupational physical activity among employed adults United States, 1990. MMWR, 49:420-424.

POWELL, K. E.;THOMPSON, C. J.; CASPERSEN, C. J. \& KEN-DRICK, J. S., 1987. Physical activity and the incidence of coronary heart disease. Annual Review of Public Health, 8:253-287.

SICHIERI, R.; COUTINHO, D. C.; LEÃO, M. M.; RECINE, E. \& EVEHART, E., 1994. High temporal, geographic, and income variation in body mass index among adults in Brazil. American Journal of Public Health, 84:793-798.

SICHIERI, R., 1998. Epidemiologia da Obesidade. Rio de Janeiro: Eduerj.

RESEARCH TRI ANGLE INSTITUTE, 1991. Softwarefor Survey Data Analysis (SUDAAN), Version 5.40. Research Triangle Park: Research Triangle Institute.

U.S. PREVENTIVE SERVICE TASK FORCE, 1996. Guide to Clinical PreventiveServices. 2nd Ed. Baltimore: Willians \& Wilkins.

WILLETT, W., 1998. Nutritional Epidemiology. 2nd Ed. Oxford: Oxford University Press.

YUSUF, H. R.; CROFT, J. B.; GILES, W. H.; ANDA, R. F.; CASPER, M. L.; CASPERSEN, C. J. \& JONES, D. A., 1996. Leisure-time physical activity among older adults. Archives of Internal Medicine, 156:13211326.

WHO (World Health Organization), 1997. Obesity: Preventing and Managing the Global Epidemic. Report of a WHO Consultation on Obesity. Geneva: WHO. 